\title{
Lived Experiences of Persons with Chronic Schizophrenia Living in the Community
}

\author{
Akanksha Rani $^{*}$, Sphoorthi G. Prabhu ${ }^{1}$, Janaki Raman ${ }^{1}$, Sojan Antony ${ }^{1}$, Thirumoorthy \\ Ammapattian $^{1}$ \\ ${ }^{1}$ Psychiatric Social Worker, Department of Psychiatric Social Work, National Institute of Mental \\ Health and Neurosciences, Bengaluru, India.
}

\begin{abstract}
Background and Purpose: Schizophrenia is a chronic and severe mental disorder which impacts a person's ability to successfully function in the community. When it interacts with structural and situational stress like poverty, homelessness and unemployment, it can lead to negative experiences and makes a person vulnerable to abuse or even develop resilience or retaining a meaningful life within the limitation of the disorder. The study aims to understand how individuals diagnosed with schizophrenia define and evaluate their experience of living in the community; personal and environmental strengths, impact of illness on their personal and social life, the challenges and barriers they meet in their day to day life and how they overcome those challenges.
\end{abstract}

Methods: The study focuses on lived experiences and deriving meaning from those experiences from a service user perspective. Therefore, Hermeneutic phenomenological approach will be used. In-depth interviews will be conducted over the course of four months to elicit client's narratives of their experiences. The interviews are transcribed, read and coded to cluster thematic aspects in each case by using ATLAS.ti.v.7. Data will be collected till saturation point is reached and participants are from various age-group, socio-economic status, ethnicity and educational background, living in the Community.

Implication: Participants' stories would narrate sources of strength, process of normalization, describing instances of discrimination, social and structural factors which they encounter affecting their help-seeking behaviour and how these factors act as facilitator and barriers in their day to day life. Findings would suggest the need for advocacy services which are discussed through recommendations and suggestions.

Keywords: Schizophrenia, Lived Experience and Community Care

\section{Background}

Schizophrenia is a chronic and severe mental disorder which affects around 21 million people worldwide, most of them living in developing countries (World Health Organisation, 2013).

\footnotetext{
*Correspondence to Akanksha Rani, Department of Psychiatric Social Work, Govindswamy Building ( $2^{\text {nd }}$ Floor), Hosur Road, Near Bangalore Milk Dairy, Bangalore- 560 029, India. Email: akanksha.rani89@gmail.com
} 
Severe and chronic nature of schizophrenia results into limited reality testing, impaired judgement, planning difficulties, difficulty in social relationships and pervasive impairment in their functionality, change the affected person's relationship with others and the social consequences are manifested in the form of discrimination, stigma and deprived opportunities. It results from negative stereotypy and prejudices that exist in society by labelling persons with the diagnosis of schizophrenia. Once labelled as suffering from schizophrenia, they are further isolated from others in ordinary day to day life. The isolation can further worsen their health status. Therefore, re-integration of the persons with schizophrenia to the community is imperative. This develops a sense of belongingness and identity. Sense of community is related to increased psychological well-being of persons with schizophrenia. Community connectedness increases their participation in community activities (Schinnar et al., 1990; Sonn \& Fiser, 1996; Rggeri et al., 2000). Townley (2012) has stated that participation in community life positively impacts health. However, patients could be doubting their ability to reintegrate to the community. So, it's important to prepare persons with schizophrenia to reintegrate back to the community after hospitalization. Therefore, need based supportive services which would help them relearn skills required for effective community integration are considered to be beneficial (Prezza et al., 2001; Cook \& Jonikas, 2002). So, while preparing for their transition into the community, concrete strategies for attaining long-term rehabilitation goals to view return to the community as an opportunity to start over as a functional member of the community, is emphasised and developed by professionals. Although there is evidence to suggest positive outcome of community re-integration for persons with schizophrenia and various strategies are developed for preparing them for the same, there are significant challenges like lack of opportunities to fulfil meaningful roles and activities in their communities, stigma and discrimination which further marginalized persons with Schizophrenia from mainstream society (Sonn \& Fisher, 1996; Prezza et al., 2001).

The persons with schizophrenia are common victims of abused which is a serious public health concern. The widespread stigma related to mental illness has increased the vulnerability of person with Schizophrenia towards abuse (Lysaker, Outcalt \& Ringer, 2010). Abuse in response to their mental illness could lead to poor outcome of illness (Lorencz, 1991). Moreover, in most of the cases, the patients would have already experienced traumatic events prior to the development of the illness, like childhood sexual abuse, physical abuse, serious neglect which often led to development of psychotic symptoms later on in life (Townley, 2012). with regards to abuses after the development of illness, research has shown that women are more likely to experience abuse from an intimate partner where as men are more likely to experience abuse perpetrated by a stranger or acquaintance. But factors which influences abuse remains more or less the same for both the genders (Wong \& Solomon, 2002; Bromley et al., 2013). Thus, it can be noted that the experiences of discrimination among persons with Schizophrenia are diverse and are determined by a number of factors. The diverse experiences of persons with Schizophrenia, factors for the same and ways to enhance positive experiences are discussed in the next session on literature review.

\subsection{Review of the literature}

Multiple studies have reported that experience of living in the community involved being stigmatized by their families, friends, landlords, police officers and community members that often led to the feelings of alienation, shame, isolation, rejection and distrust which resulted in social isolation and discrimination which marginalised them further. Another study has discussed the perceived reason for discrimination was person's mental illness rather than

Social Science Protocols, Sepetmber 2019, 1-12.

http://dx.doi.org/10.7565/ssp.2019.2656 
other characteristics associated with stigma such as race, gender, sexual orientation. The instances of discrimination experienced by participants were in the form of difficulty in finding jobs, house for rent and development of sense of injustice because of being treated or judged negatively by law enforcers like police officers and community members. The authors concluded that health care professionals had a significant role in reducing stigma through counselling and community-based awareness programmes (Cook\& Jonikas, 2002; Bond, 1998; Murphy, 2008; Bromley et al., 2013; Wong, Sands \& Solomon, 2010; Pahwa \& Kriegel, 2018). Likewise, Borg \& Davidson (2008) assessed experience of illness of persons with SMI, its consequences into their day to day lives and how they derive sense of meaning from those experiences. The authors reported that for persons with illness like Schizophrenia, being normal meant being able to spend time with ordinary people in their community. Being positive created good feelings and keeping busy, by engaging in pleasurable recreational activities, helped them to develop sense of belonging in the community and positive meaning of their experiences. Effective community integration depends upon socio-cultural context. Keeping in mind the social norms and culturally favoured activities are encouraged which helps persons with Schizophrenia to derive positive meaning of their experience and recover fast.

There has not been much research on persons with Schizophrenia's positive experience of living in the community. Few studies have discussed how positive past experiences gives participants opportunity to respond generatively by giving back (altruism) which restores hope in them even in most difficult circumstances. One of the studies highlights the importance of harnessing persons existing strengths and abilities which is not affected by their illness and which can also lead to skills development, effective rehabilitation and reintegration into the community (Corrigan et al., 2005; Gunnmo \& Fatouros, 2011). Studies have also been collected to understand the perspective on 'experiencing community' by patients with mental illness. The authors report that for people with mental illness like schizophrenia, certain people or environment signifies community which can be certain geographical area, group homes or online support groups. The sense of belongingness and connectedness will depend upon how much persons with Schizophrenia receive help and support, which gives them opportunity to identify with others. Most respondents described their symptoms being a barrier for effective community integration as they worried that their symptoms will become threatening or irritating to others. Hence, they socially isolated themselves or socialized with only familiar people (Borg \& Davidson, 2008). As far as factors associated with differential experiences are concerned, several studies have highlighted socio-cultural factors having a significant role to play. Women are socialized in being passive. Their roles included being nurturing, supportive and are expected to perform their roles and responsibilities as a wife, mother or daughter. It was also noted that they have higher motivation level to recover fast. But women are also vulnerable to gender-based violence if they are not able to perform their roles and responsibilities adequately post illness (Cummins \& Lau, 2003; Desai et al., 2016).

With regards to ways to enhance positive experiences, multiple studies have reported that persons with severe mental illness like Schizophrenia needs supportive socialization, assistance to grieve losses, conflict resolution skills for managing potential conflicts with family members and need to engage in productive work. It will give a sense of accomplishment and improve their self-esteem, activities of daily living and social life. Supported employment and educational programs would act as an incentive in helping persons with Severe mental illnesses to derive positive meaning of their experience of living with severe mental illness (Silver, 2002; Shrivastava \& Shrivastava, 2013; Stevens et al., 2013).

Social Science Protocols, Sepetmber 2019, 1-12.

http://dx.doi.org/10.7565/ssp.2019.2656 


\subsection{Knowledge gap}

In summary, review shows that research is dominated by high-income countries, with most studies done in the USA and the UK, as these countries increased awareness towards mental health care needs has led to providing quality care through social security and communitybased service delivery. Most of these studies has been quantitative in nature or secondary data analysis rather than exploring qualitatively through in-depth interviews and narratives. Most of these studies have focused on negative aspect of living with schizophrenia by trying to understand their experience of being discriminated, marginalized and isolated but there is also need to explore factors which helped to build resilience or retaining a meaningful life within the limitation of the disorder.

\subsection{Need for the study}

In India, there is poor quality of care due to shortage of human resources, infrastructure, government budget cuts and service delivery system which is limited to periphery rather than community based. There is also lack of community-based follow up studies to explore experiences of persons with schizophrenia after reintegrating them into community. Persons with schizophrenia feels isolated and marginalised due to negative stereotypes and prevalent stigma related to mental illness which act as a barrier in their effective community integration (Murphy, 2008).

Multiple studies have shown that the meaning of social integration is different for different people and even though persons with scizophrenia may live in community with ordinary people, they remain socially isolated (Wong, Sands \& Solomon, 2010; Bromley et al., 2013). Studies have also shown that community life can be full of challenges as well as opportunities. When these interact with structural and situational stress like poverty, homelessness and unemployment, it can lead to negative experiences or buffer the effects of those experiences by making them vulnerable to abuse or developing resilience or ability to protect themselves from abuse (Pahwa \& Kriegel, 2018; Cook, 1994; McCann \& Clark, 2004).

Each individual with mental illness experience life uniquely despite the sameness suggested by universal labelling of person as mentally ill. To better understand the diverse subjective experience in terms of strengths, opportunities and barriers, a qualitative examination of their narratives would be required which will be done by the current study. Present study would also try to explore ways in which individuals diagnosed with schizophrenia define and evaluate their experience of living in the community, personal and environmental strengths, impact of illness on their personal and social life, the challenges and barriers they meet in their day to day life and how they overcome those challenges.

\subsection{Scope of the study}

- This study would help us to understand the subjective experience of having schizophrenia and deriving meaning of those experiences from a service user perspective. It will also provide us with deeper and more realistic understanding of the complexity of living and experiencing mental illness like schizophrenia and how contextual factors- personal and environmental factors acts as a barrier or facilitator.

- It is important to explore need for interventions from service user perspective and design client centred intervention programme which is cultural specific and indigenous in nature with community-based service delivery model which is led by service user.

Social Science Protocols, Sepetmber 2019, 1-12.

http://dx.doi.org/10.7565/ssp.2019.2656 


\subsection{Aim}

To study lived experiences of Persons with Schizophrenia living in the community.

\subsection{Objectives}

1) To assess subjective experience of having schizophrenia and deriving meaning of those experiences

2) To assess how personal and environmental factors acts as a barrier or facilitator

3) To assess impact of illness on their personal and social life

4) To compare the subjective experience of having schizophrenia among men and women

\section{Operational Definitions}

a) Chronic schizophrenia refers to severe mental disorder which is characterized by recurrent and/or persistent features affecting a person's thoughts, beliefs, feelings and behaviour leading to bio-psycho-social dysfunction; the diagnosis which is made as per ICD-10 criteria and measured using validated diagnostic instrument or clinical records. As the participants need to have a diagnosis long enough to be able to describe impact of illness on his/her life, a minimum illness duration of one year and those who have been hospitalized for more than twice have been considered as having chronic schizophrenia.

b) Lived experiences

It's personal and unique perspective of participants' experience of having Schizophrenia and how their experience, strengths and abilities get influenced by feelings, thoughts, behaviour, and how contextual factors- personal and environmental factors-act as a barrier or facilitator. These factors would either help to build resilience or to retain a meaningful life within the limitation of the disorder or would act as a risk factor for domestic violence, discrimination or stigma.

\section{c) Community}

The neighborhood where participants are currently living along with their family members. It also comprises of physical and social conditions of their neighborhood in the form of living space, resources available and accessible, utility of public places, attitude of community people towards them and materialistic and social support provided by the community people.

\section{d) Barriers and facilitators}

Barriers to effective community integration may include poverty, unemployment, homelessness, financial constraints, denial of opportunity to participate in civic and community life, unsupportive working conditions, public places, transportation, social functions and job opportunities being denied or not accessible and stigma related to mental illness; thus limiting the opportunities for meaningful integration into their local and extended community and results into social avoidance and exploitation. Facilitators reduces person's disability caused due to mental illness and on the other hand enhances the level of functioning leading to increase social participation. It may include developing sense of self-efficacy, resourcefulness, resilience, inclusive attitude, provision of services for persons with schizophrenia, accessibility to community resources and technological devices and social support. 


\section{e) Personal \& environmental factors}

Personal factors which may include socio-demographic details (age, gender, ethnicity, social and educational background, job opportunities), life-events, personal strengths, deficits and psychological factors like reaction pattern to stress, coping or problemsolving skills and sense of belongingness to the community. Environmental factors may include geographical location, accessibility to community resources (social welfare benefits, employment agencies, religious institute, legal system), positive and negative attitude of community people which increases or decreases social participation of persons with Schizophrenia.

\section{Study Design}

It is Exploratory Research Design. The study focuses on lived experiences and deriving meaning from those experiences from a service user perspective. Therefore, Hermeneutic phenomenological approach will be used. Creswell \& Creswell (2017) has argued that Hermeneutic approach states that every phenomenon has many possible perspectives and understanding of a phenomena is not about accurately describing or deriving pre-existing meaning but the meaning is created by trying to understand the phenomena (Cook, 1994). So, focus is on contextuality and situatedness. According to Gadamer (1975) every person experiences the world in a way that is unique and personally meaningful which is rooted within the context of his or her individual history. The meaning of phenomena is generated through dialogue in social relations as words and stories are shared in common as an inter-state subjective discourse.

\subsection{Setting}

Persons with schizophrenia staying in the community and coming for follow-up to the outpatient psychiatry department at NIMHANS, Bengaluru. Data collection will be done between September to October, 2019. It will be one time recruitment of patients without follow-ups.

\subsection{Study population}

Persons with Schizophrenia, residing in communities with their families coming for follow up at the out-patient psychaitry department of NIMHANS will be selected and will be considered as representative of persons with Scizophrenia residing in community.

\subsection{Sampling}

Participants satisfying the inclusion and exclusion criteria will be selected through purposive sampling. In-depth interviews would be conducted with 15-20 participants (both men and women) till data saturation is reached, where no new findings emerge.

\subsection{Inclusion criteria}

- Persons with a diagnosis of chronic Schizophrenia Spectrum Disorder according to ICD-10 criteria;

- Persons with Schizophrenia with a minimum illness duration of one year and who have been hospitalized more than twice;

- Those who are maintaining well based on CGI score $(\leq 4)$ and receiving adult psychiatric services from NIMHANS on outpatient basis;

Social Science Protocols, Sepetmber 2019, 1-12.

http://dx.doi.org/10.7565/ssp.2019.2656 
- Persons who are 18 years or above and of any gender;

- Persons with Schizophrenia who are staying in community for at least 6 months

- Persons who can speak Hindi, English and Kannada and give written informed consent.

\subsection{Exclusion criteria}

- Persons who have Intellectual Developmental Disorder as per standard assessments;

- Persons having formal thought disorder;

- Persons who are unable to give written informed consent to participate in the study;

- Persons with repeated history of Epilepsy and Neurodegenerative Disease like Parkinson, Alzheimer and Dementia.

\subsection{Tools for data collection}

\section{1) Semi-structured interview schedule}

It will be divided into two parts:

\section{Part A: Socio-demographic data sheet}

It will be prepared by the research team and will include personal data like age, gender, marital status, educational status, details about family of origin and if married then details about family of procreation, duration of staying in residential rehabilitation home, reasons for enrolment.

\section{Part B: Clinical profile}

Clinical profile will include details about illness like onset, duration of illness, precipitating factor, course, brief history, current symptoms, pre-morbid personality.

\section{2) Symptoms severity: Clinical Global Impression Scale (CGI)}

It is a 3-item observer-rated scale that measures illness severity (CGIS), global impression or change (CGIC) and therapeutic response. The CGI is rated on a 7 point scale, with the severity of illness scale using a range of responses from 1 (normal) through to 7 (amongst the most severely ill patients). The scale is in public domain.

\section{3) In-depth interview guide}

An in-depth interview will be conducted to collect information pertaining to lived experience of persons with Schizophrenia. The guide will be prepared by the research team for in-depth interviews which will be based on literature and experts' opinion. The in-depth interview will be following a semi-structured format, using open-ended questions, in a face to face conversational style. It will cover the key topics which will be explored with the respondents. The interview guide will be flexible in nature but some direction would be given by using probes whenever necessary so that focus is not lost. Some of the areas which would be covered are subjective experience of discrimination, influence of gender on discrimination and social and structural barriers which decreases momentum of community integration. Face and content validation of interview guide will be done.

\section{Methods of Data Collection}

\subsection{In-depth interviews}

Hermeneutic dialogue and interviewing approach emphasize on openness and nondirectiveness during the course of interview. So, the researcher will actively pose open-ended 
questions. Hermeneutic approach treats both researcher and participants as equal partners and both the parties play a complimentary role in generating and understanding the experiences and meaning derived from those experiences.

\section{Phases and Timeframe of the Study}

The study has four phases: Preparatory, Recruitment, Assessment and Data Analysis (see Figure 1).

i. Preparatory: This involves seeking consent from the ethical committee. In-depth interview guide and socio-demographic schedule would be prepared. Interview guide would be given to experts for face and content validation and their suggestions would be incorporated. Then it will be pretested with few participants.

ii. Recruitment: Persons with chronic Schizophrenia coming for psychiatric follow- up on outpatient basis to Adult Psychiatry of NIMHANS. Participants would be contacted and explained face to face regarding the nature of the study and informed consent would be obtained. Symptoms severity would be assessed through CGI and based on CGI score $(\leq 4)$ it would be decided whether persons can participate in the study or not. Data would be collected and simultaneously analysed (details explained below corresponding to in serial number 4). Participants would be recruited till data saturation is achieved.

iii. Assessment: Socio-demographic tool would be administered to eligible candidates. Qualitative interviews would be conducted by following in-depth interview guide. All interviews would be audio recorded and transcribed.

iv. Data Analysis

The data collected will be recorded as first-person narratives (Kannada, Hindi and English). All interviews will be conducted in complete privacy. Interview will be planned in single session of 45 minutes to 60 minutes and in certain cases interview may extend to two sessions. The narratives will be translated into English using ATLAS.ti.v software. Data will be analyzed through thematic content analysis. All transcribed interview will be treated as texts and then analysis will be carried out by following the principles of hermeneutic circle which states that analysis should function at two levels: the descriptive one relating to the individual parts of the phenomenon and the interpretative one that places the parts within the whole of the experience (McCann \& Clark, 2004; Creswell\& Creswell, 2017).

The analysis will be carried out at the four stages. In the first stage, the transcripts will be read and re-read repeatedly to gain overall impression of the data. In the second stage, individual transcripts will be analyzed for key themes that emerged from the data. The third stage will involve reflection on the themes as a part of the whole, which is seeing inter-theme connectivity with overall experience of the phenomenon, with relevant literature and personal reflection as recorded by the researcher. In the final stage, the themes identified for each participant will be synthesized into a coherent picture of the phenomenon including quotations to illustrate each theme (Gadamer, 1975; Laverty, 2003). 
Figure 1. Process of the Study

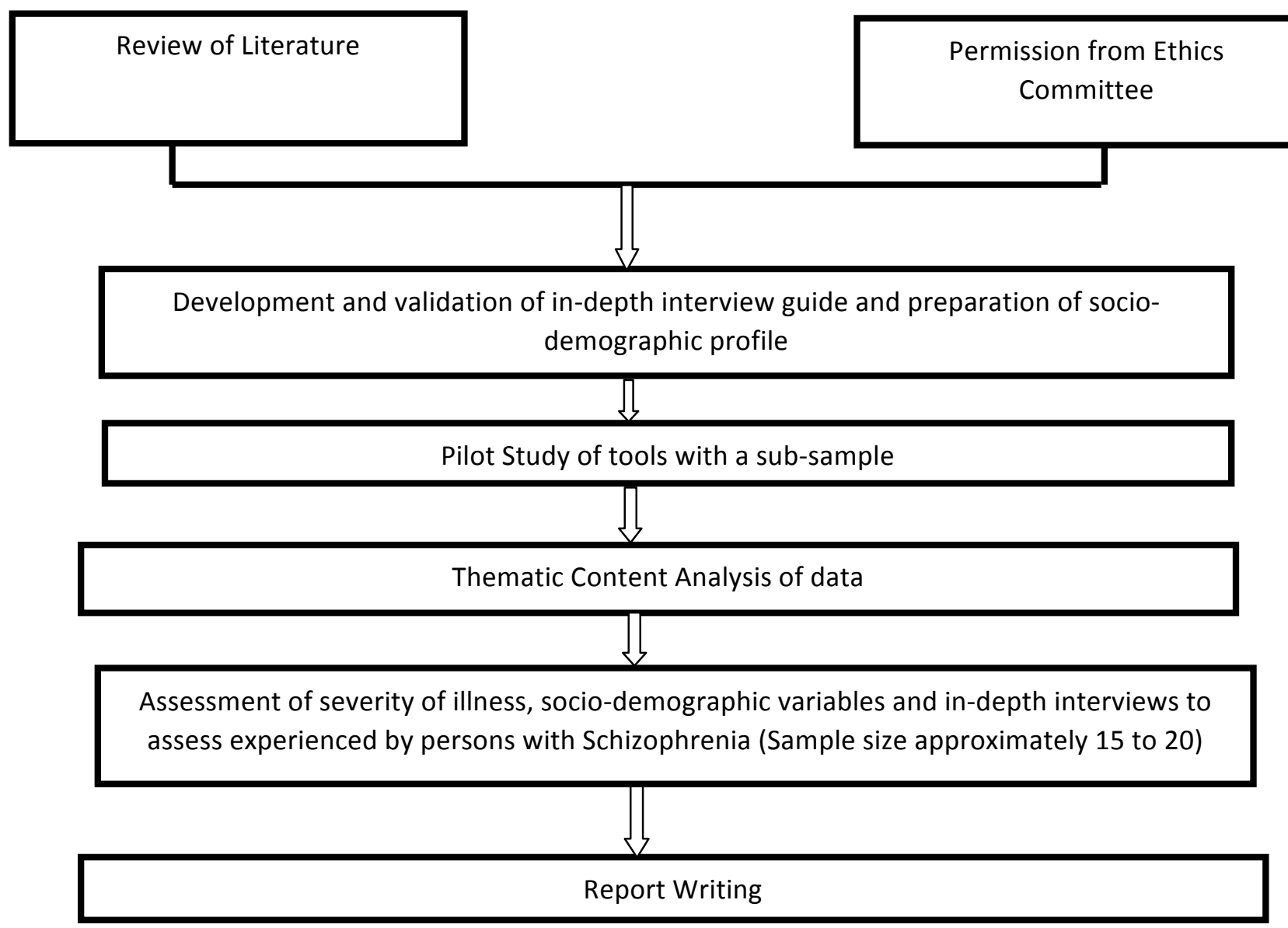

Tentative Time Framework of the Study: Figure 2 represents tentative time required (in months) and steps which will be followed to conduct the research study.

Figure 2. Time Framework of the Study

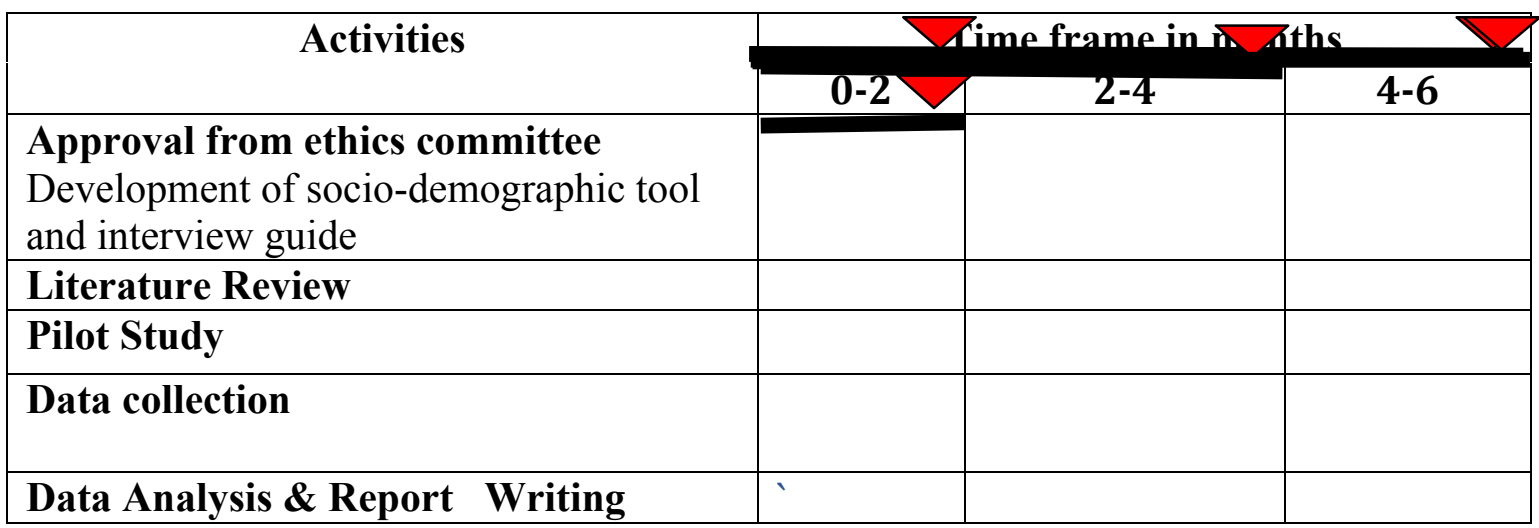

\section{Ethical consideration}

Institute Review Board (IRB) clearance was sought for the study [No. NIMHANS/EC(BEH.SC.DIV.)/2019]. Written informed consent will be obtained from the 
caregivers and participants residing in the community. The study procedure will be clearly explained to the participants and the voluntary nature of participation. The participants' confidentiality will be ensured and maintained in all stages of the study. There would be no financial benefits, traveling allowances or gifts for the participation in the study. The participants will be ascertained freedom to withdraw from the study at any point of time that withdrawal will not affect their treatment in any ways.

If there is any risk of abuse and threat to participants' safety then referrals will be provided after discussing with the treating doctor. If participant become psychologically quite distressed during the interview it will be addressed accordingly during or after the interview. Referrals will be based on participant's needs (e.g. if abuse has been identified and you or your family are in crisis during any of the phases of the study). There are different types of services which can be provided through referrals like individual counseling for emotional support, family counseling for relationship problems, information through legal aid clinic regarding legal options available to participants' or list of organizations which can help them by providing psychological, social or legal support they require. Incidental cost (e.g. coming for subsequent session post referrals) will be borne by the participants.

\section{Implication for Psychiatric Social Work Practice}

When working with vulnerable population like persons with Schizophrenia, a Psychiatric Social Worker needs to adopt social justice perspective by highlighting unequal treatment meted out to them and giving voices to those who have been marginalized by making them research partner. In this study, persons with chronic schizophrenia are study participants and client's narratives of their experiences are going to be studied. Narratives will mostly focus on sources of strength, process of normalization, social and structural factors which they encounter affecting their help-seeking behaviour. It will also try to assess how these factors act as facilitator and barriers in their day to day life.

Qualitative studies involving people with severe, persistent and chronic mental illness like schizophrenia should describe their experiences in a way that highlights how culturally and socio-economically specific contextual factors leads to discrimination and stigma or builds resilience and modifies the stigmatizing experience. The findings of the study should be synthesized in a way that it critically analyzed the phenomena and recommend strategies to improve living conditions in the community for these people.

\section{Declarations}

Funding Sources: This research did not receive any specific grant from funding agencies in the public, commercial, or not-for-profit sectors.

Conflict of interests: The authors declare that they have no conflict of interest.

\section{References}

Bond, G. R. (1998). Principles of the individual placement and support model: empirical support. Psychiatric Rehabilitation Journal, 22(1), 11.

Borg, M., \& Davidson, L. (2008). The nature of recovery as lived in everyday experience. Journal of Mental Health, 17(2), 129-140. 
Bromley, E., Gabrielian, S., Brekke, B., Pahwa, R., Daly, K. A., Brekke, J. S., \& Braslow, J. T. (2013). Experiencing community: Perspectives of individuals diagnosed as having serious mental illness. Psychiatric Services, 64(7), 672-679.

Cook, J. A. (1994). Independent community living among women with severe mental illness: A comparison with outcomes among men. The Journal of Mental Health Administration, 21(4), 361-373.

Cook, J. A., \& Jonikas, J. A. (2002). Self-determination among mental health consumers/survivors: Using lessons from the past to guide the future. Journal of Disability Policy Studies, 13(2), 88-96.

Corrigan, P. W., Slopen, N., Gracia, G., Phelan, S., Keogh, C. B., \& Keck, L. (2005). Some recovery processes in mutual-help groups for persons with mental illness; II: Qualitative analysis of participant interviews. Community Mental Health Journal, 41(6), 721-735.

Creswell, J. W., \& Creswell, J. D. (2017). Research design: Qualitative, quantitative, and mixed methods approaches. London: Sage.

Cummins, R. A., \& Lau, A. L. (2003). Community integration or community exposure? A review and discussion in relation to people with an intellectual disability. Journal of Applied Research in Intellectual Disabilities, 16(2), 145-157.

Desai, R., Abraham, D., Harshe, D. G., Ramakrishnan, A., Cholera, R., \& Kale, S. S. (2016). A study of depression, perceived loneliness, cognitive function and independence in daily activities in home and institution based older people: A cross sectional comparative study. Journal of Geriatric Mental Health, 3(2), 140.

Gadamer, H. G. (1975). Hermeneutics and social science. Cultural hermeneutics. Philosophy and Social Criticism, 2(4), 307-316.

Gunnmo, P., \& Fatouros Bergman, H. (2011). What do individuals with schizophrenia need to increase their well-being. International Journal of Qualitative Studies on Health and Well-Being, 6(1), 5412.

Laverty, S. M. (2003). Hermeneutic phenomenology and phenomenology: A comparison of historical and methodological considerations. International Journal of Qualitative Methods, 2(3), 21-35.

Lorencz, B. (1991). Becoming ordinary: Leaving the psychiatric hospital. The illness experience: Dimensions of suffering. London: Sage.

Lysaker, P. H., Outcalt, S. D., \& Ringer, J. M. (2010). Clinical and psychosocial significance of trauma history in schizophrenia spectrum disorders. Expert Review of Neurotherapeutics, 10(7), 1143-1151.

McCann, T. V., \& Clark, E. (2004). Embodiment of severe and enduring mental illness: Finding meaning in schizophrenia. Issues in Mental Health Nursing, 25(8), 783-798.

Murphy, M. (2008). Residential placement and well-being among persons recovering from serious mental illness, Doctoral Dissertation, Boston College, Graduate School of Social Work.

Pahwa, R., \& Kriegel, L. (2018). Psychological community integration of individuals with serious mental illness. The Journal of Nervous and Mental Disease, 206(6), 410-416.

Prezza, M., Amici, M., Roberti, T., \& Tedeschi, G. (2001). Sense of community referred to the whole town: Its relations with neighbouring, loneliness, life satisfaction, and area of residence. Journal of community psychology, 29(1), 29-52.

Ruggeri, M., Leese, M., Thornicroft, G., Bisoffi, G., \& Tansella, M. (2000). Definition and prevalence of severe and persistent mental illness. The British Journal of Psychiatry, 177(2), 149-155.

Social Science Protocols, Sepetmber 2019, 1-12.

http://dx.doi.org/10.7565/ssp.2019.2656 
Schinnar, A. P., Rothbard, A. B., Kanter, R., \& Jung, Y. S. (1990). An empirical literature review of definitions of severe and persistent mental illness. The American Journal of Psychiatry.

Shrivastava, P. S., \& Shrivastava, S. R. (2013). A study of spousal domestic violence in an urban slum of Mumbai. International journal of Preventive Medicine, 4(1), 27.

Silver, E. (2002). Mental disorder and violent victimization: the mediating role of involvement in conflicted social relationships. Criminology, 40(1), 191-212.

Sonn, C. C., \& Fisher, A. T. (1996). Psychological sense of community in a politically constructed group. Journal of Community Psychology, 24(4), 417-430.

Stevens, M., Biggs, S., Dixon, J., Tinker, A., \& Manthorpe, J. (2013). Interactional perspectives on the mistreatment of older and vulnerable people in long-term care settings. The British Journal of Sociology, 64(2), 267-286.

Teasdale, B. (2009). Mental disorder and violent victimization. Criminal Justice and Behavior, 36(5), 513-535.

Townley, G. E. (2012). Examining community integration of individuals with psychiatric disabilities residing in supported and non-supported housing, Doctoral Dissertation, University of South Carolina.

Wong, Y. L. I., \& Solomon, P. L. (2002). Community integration of persons with psychiatric disabilities in supportive independent housing: A conceptual model and methodological considerations. Mental health Services Research, 4(1), 13-28.

Wong, Y. L. I., Sands, R. G., \& Solomon, P. L. (2010). Conceptualizing community: The experience of mental health consumers. Qualitative Health Research, 20(5), 654-667.

World Health Organization. (2013). Investing in mental health: evidence for action. Geneva, Switzerland. World Health Organization. 